\title{
Réaliser des équipements hydrauliques silencieux
}

\section{Producing silent hydraulic equipment}

\author{
J. Delcambre \\ Direction des Etudes \\ et Recherches
}

\author{
J. Rabaud \\ Direction \\ de l'Equipement
}

\section{G. Vuillerod M. Wegner}

Société Neyrpic

Grenoble

Electricité de France

La conception et la réalisation des équipements hydrauliques comportent de nombreuses contraintes parmi lesquelles le bruit qui peut, aujourd'hui, être mieux pris en compte. Après l'analyse d'un cas typique, les auteurs montrent que l'étude acoustique préalable de ces ouvrages, basée sur une définition des zones à protéger du bruit des machines les plus bruyantes, permet d'obtenir des résultats positifs pour un surcoût réduit.

Les efforts conjoints du Constructeur et du Mâtre d'Euvre, en même temps que le développement des outils modernes de l'acoustique prévisionnelle, permettent d'espérer une précision de plus en plus grande dans la conception d'équipements hydrauliques silencieux.

One of the many constraints facing the design and construction of hydraulic equipment is noise which can now be better accommodated. After an analysis of a typical case, the authors demonstrate that the prior acoustic study of these works, based on a definition of the zones to be protected from the noisiest machines, enables positive results to be obtained at a reduced excess cost.

The joint efforts of the Constructor and the Cheif Architect, together with the development of modern tools in provisional acoustics, allow one to anticipate an even greater precision in the conception of hydraulic silent equipment.

1. Une expérience typique : La centrale d'Auzat

Lors de la mise en service de la centrale d'Auzat, des niveaux sonores importants ont été observés sur plusieurs planchers et la décision fut prise d'améliorer cette installation, en commençant par une campagne de mesure.

Quatre objectifs étaient assignés à cette campagne conduite en février 1983 par Neyrpic et Electricité de France (DTG Grenoble) : a) compléter le diagnostic technique sur une centrale réputée bruyante,

b) étudier l'intérêt et la pratique d'isolations autour des sources sonores principales,

c) éprouver sur le terrain la rédaction des cahiers des charges actuels concernant le bruit et, en particulier, éprouver la pertinence de la norme NF S 31067 de janvier 1982 "Détermination de la puissance acoustique émise par les sources de bruit ",

d) repérer les phénomènes physiques (hydrauliques, électriques, mécaniques) contribuant aux sources de bruit. 

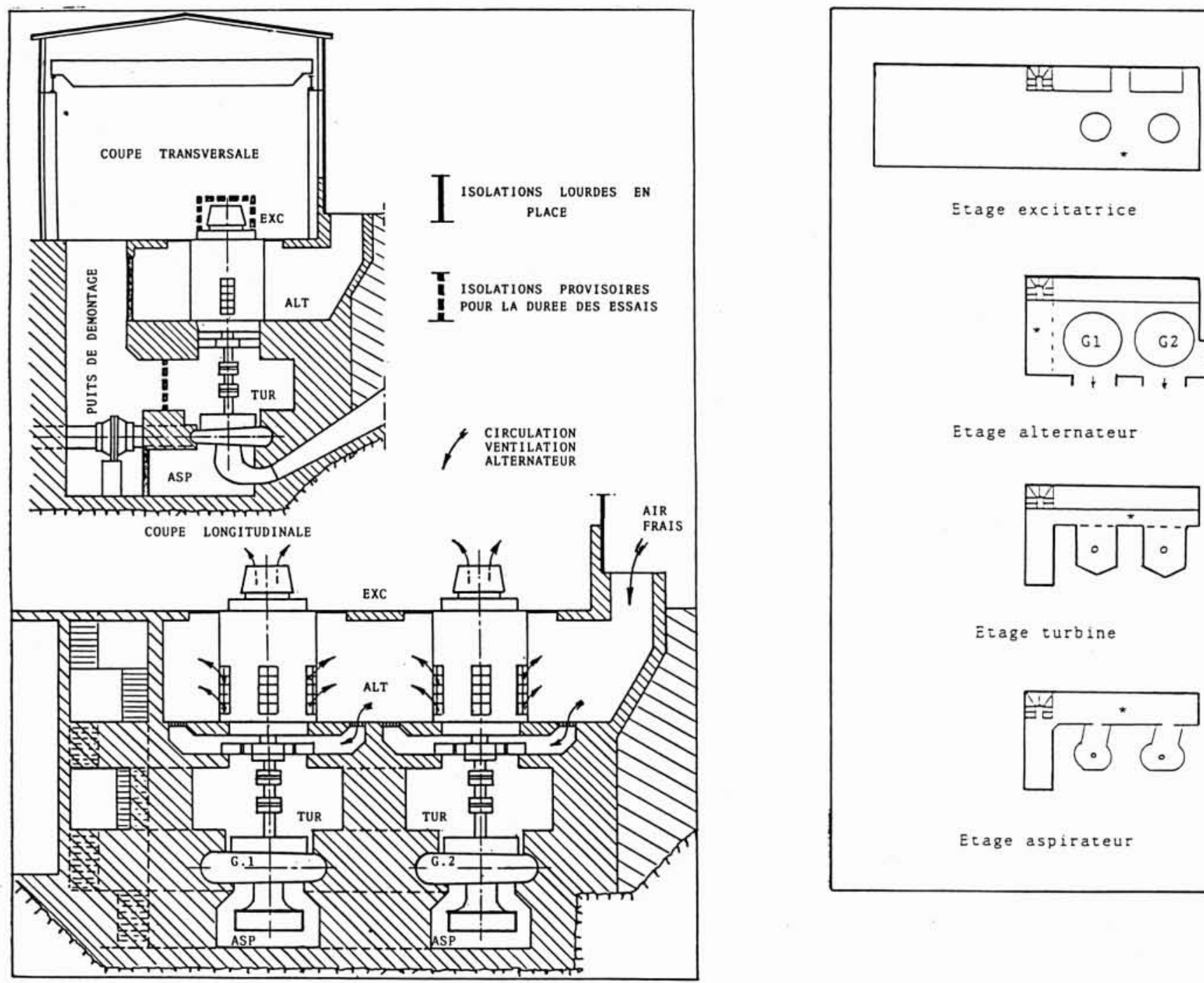

Etage excitatice

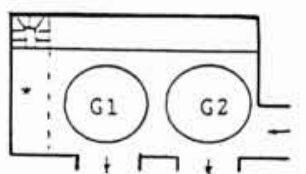

Etage alternateur

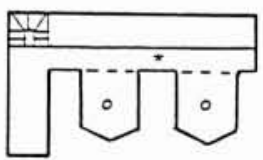

Etage turbine

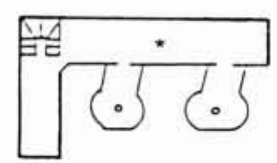

Etage aspirateur

1. Centrale d'Auzat : coupes schématiques.

2. Emplacement des isolations et des points de mesures d'ambiance.

Rappelons les caractéristiques utiles de la centrale de Auzat :

2 turbines Francis verticales

- Puissance nominale sur l'arbre

- Chute nette nominale

- Débit nominal

- Vitesse de rotation

- Diamètre de sortie de roue

- Nombre d'aubes

26,15 MW

$404 \mathrm{~m}$

$7,2 \mathrm{~m}^{3} / \mathrm{s}$

$1000 \mathrm{tr} / \mathrm{min}$

$900 \mathrm{~mm}$

- Nombre de directrices

15

20

- Nombre de pôles alternateur

- Nombre d'ailettes du ventilateur

\subsection{Processus de mesure}

Les niveaux de bruit d'ambiance ont, dans un premier temps, été collectionnés en diverses zones de la centrale où le personnel d'exploitation ou d'entretien peut se trouver impliqué. Tous les régimes de fonctionnement stabilisés ont été explorés et l'influence de plusieurs combinaisons de dispositifs isolants existants ou établis provisoirement pour les besoins des essais a été analysée. La caractérisation des spectres des quatre principales sources de bruit a ensuite été tentée sur le groupe $\mathrm{n}^{\circ} 1$ en exploitation à $17 \mathrm{MW}$ ainsi que la détermination de leur niveau de puissance acoustique suivant la norme NF S 31067.

Enfin, les corrections d'environnement selon cette norme ont été vérifiées par l'étude des temps de réverbération. 


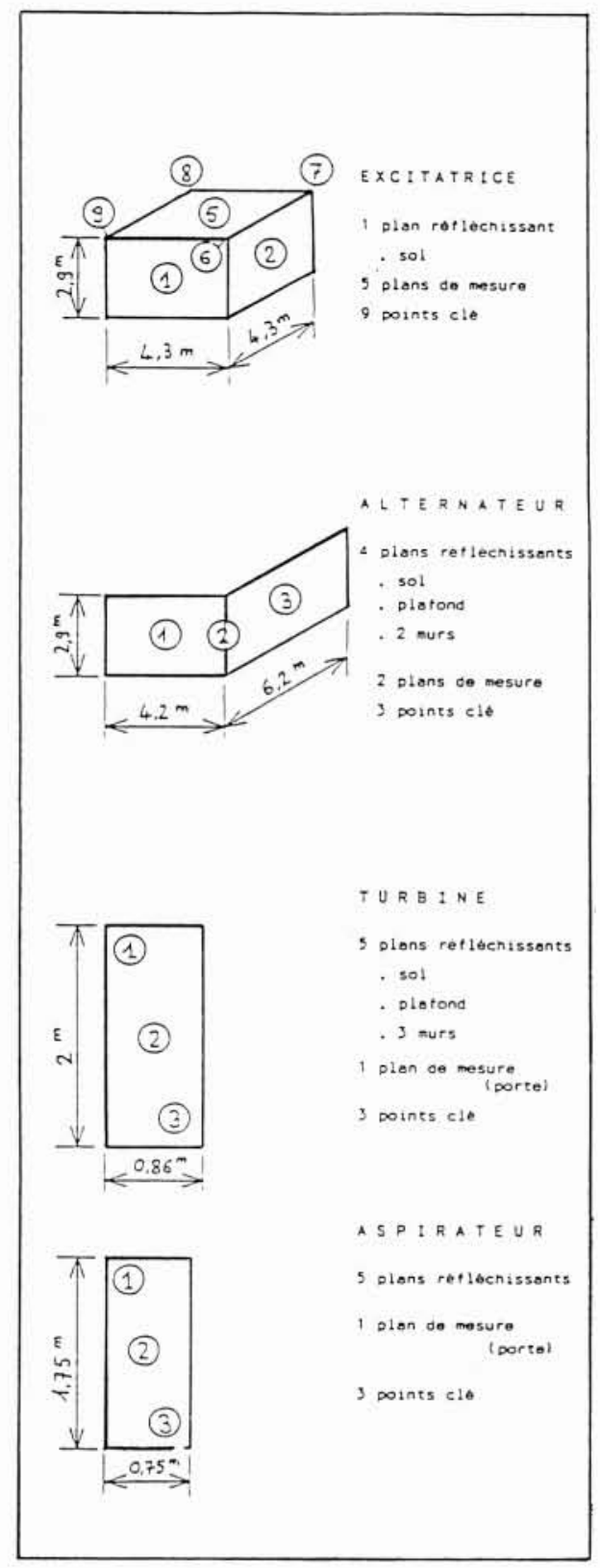

3. Caractérisation des sources selon la norme NFS 31067 . Points clés et surfaces de mesurage.

\subsubsection{Mesures d'ambiance}

La centrale d'Auzat comporte deux groupes verticaux situés côte à côte, les quatre étages techniques (excitatrice, alternateur, turbine et aspirateur) sont mis en communication par le puits de démontage de la largeur des deux groupes (fig. 1).

Le génie civil enserre les fonds supérieurs turbines et les aspirateurs dans des cavités indépendantes ouvrant sur des coursives d'accès latéral.

Une cloison phonique lourde isole conjointement les deux alternateurs dont la ventilation est commune.
L'étage supérieur inclut dans un même volume sous plafond les deux excitatrices et les tableaux de commande de la centrale.

De nombreuses mesures de niveaux de pression sonore en pondération $\mathrm{A}$ ont été effectuées pour plusieurs conditions d'exploitation du groupe 1 (le groupe 2 étant à l'arrêt) et plusieurs configurations des isolations complémentaires. Celles-ci sont discutées au §3. Quelques analyses spectrales par bandes d'octaves et tiers d'octaves ont également été conduites dans l'espoir d'identifier l'origine des bruits par comparaison aux spectres des différentes sources sonores. La figure 2 précise l'emplacement des mesures d'ambiance.

\subsubsection{Mesures des puissances acoustiques des sources}

La norme NF S 31067 préconise une méthode de détermination de la puissance acoustique des machines et équipements qui ne peuvent être déplacés hors de leur lieu d'installation pour les besoins des relevés.

Le niveau de puissance acoustique de la source de bruit est calculé d'après les valeurs mesurées du niveau de la pression acoustique et en utilisant des corrections d'environnement.

La norme définit le nombre et les emplacements des positions (points clés) du microphone sur la surface de mesurage en fonction de la taille de la source et de la disposition des plans réfléchissants. Cette surface est constituée par une ou plusieurs faces rectangulaires situées à des distances égales des faces de la surface de référence, soit « le plus petit parallélépipède rectangle enveloppant la source ".

Le niveau de pression acoustique quadratique moyen par octave est alors exprimé comme suit :

$$
\bar{L}_{p}=10 \log \left(\frac{1}{N} \sum_{i=1}^{N} 10^{0.1} L_{p i}\right)-K
$$

- Lpi est le niveau de pression acoustique obtenu à la ième mesure,

- $N$ est le nombre total de mesures,

- $K$ est le facteur de correction d'environnement

et le niveau de puissance acoustique par octave s'obtient par référence au flux au travers d'une surface So de $1 \mathrm{~m}^{2}$

$$
L_{n}=\bar{L}_{p}+10 \log (S / S o)
$$

- $S$ est l'aire de la surface de mesurage, en mètres carrés.

Le facteur correctif d'environnement $K$ est obtenu en remplaçant provisoirement la source de bruit à caractériser par une source sonore de référence (SSR) et en comparant les niveaux bruts de puissance obtenus sans correction avec ceux relevés lors de son étalonnage en laboratoire sur un plan réfléchissant.

Les figures 3 donnent une idée des surfaces de mesurage pour chacune des sources et situent les positions des points clés.

\subsubsection{Mesure des temps de réverbération}

L'application de la norme NF S 31067 dans les conditions réelles rencontrées sur site se heurte à de sérieuses difficultés pour la détermination du facteur d'environnement $K$. Nous avons étudié un autre mode d'évaluation fondé sur la mesure des temps de réverbération. 


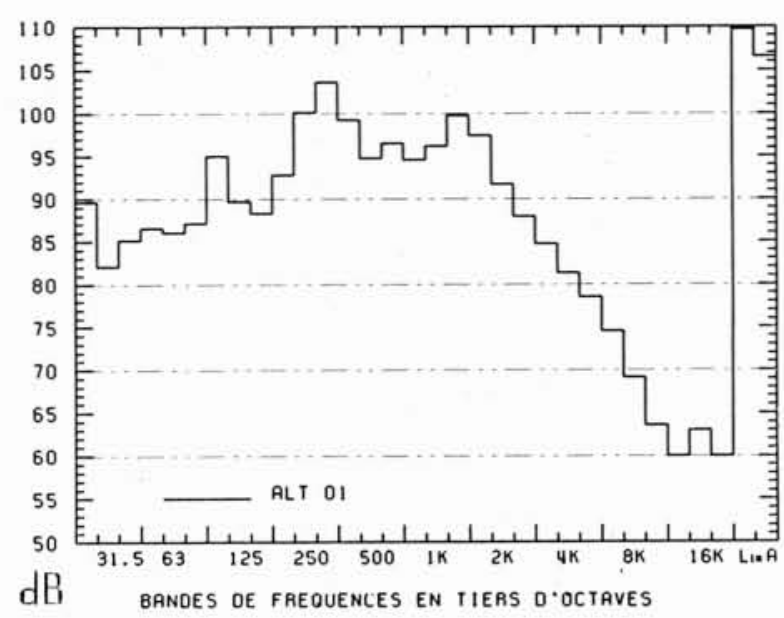

4. Alternateur : spectre au point clé $n^{\circ} 1$.

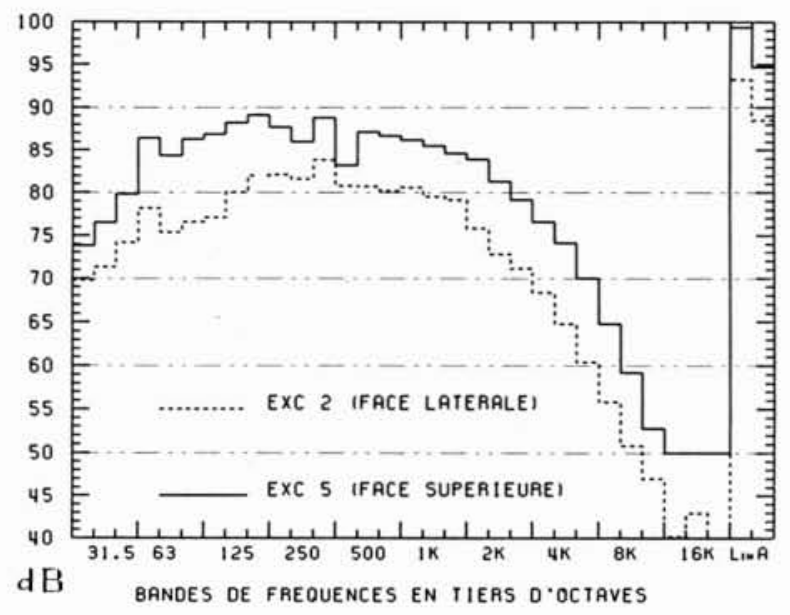

5. Excitatrice: comparaison des spectres au milieu des faces supérieure et latérale.

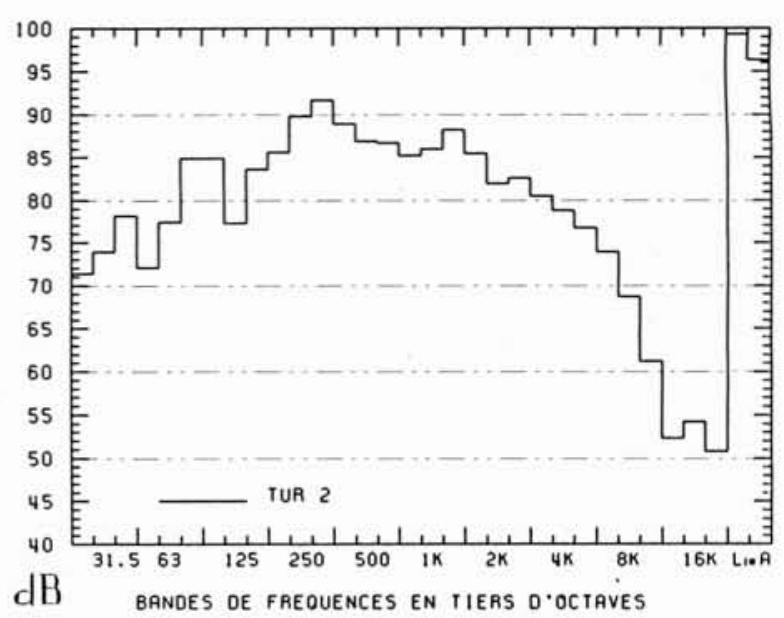

6. Etage turbine. Spectre au point clé $n^{\circ} 2$ (milieu de la porte d'accès).

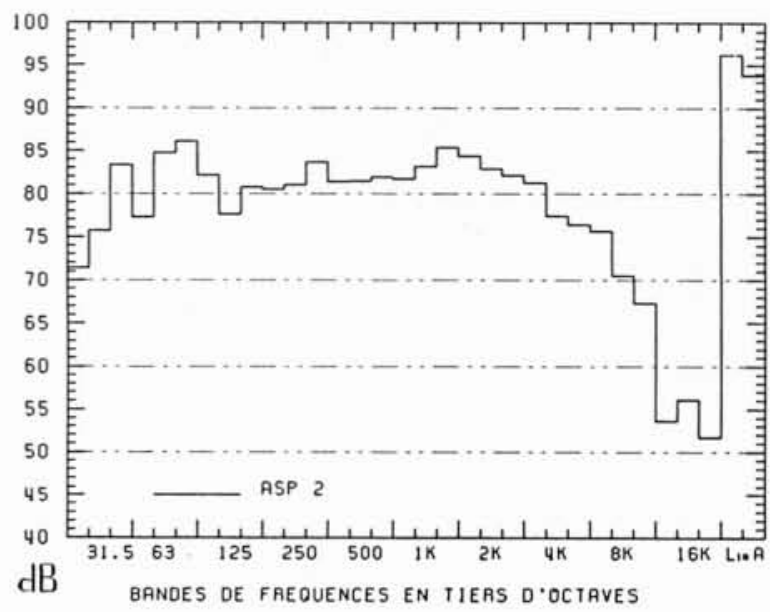

7. Aspirateur : spectre au point clé n $n^{\circ} 2$ (milieu de la porte d'accès).
Dans les instants suivant un bruit impulsif (tir au pistolet), le niveau sonore au sein du volume étudié décroît sensiblement selon une loi exponentielle due aux phénomènes d'absorption. On mesure le temps de réverbération $T$, au bout duquel le niveau moyen a décru de $60 \mathrm{~dB}$.

La loi de Sabine permet de calculer l'aire d'absorption équivalente

$$
A=0,16(V / T)
$$

et d'estimer le facteur d'environnement $K_{\text {Lin }}$

$$
K_{\text {Ln }}=10 \log \left[1+\frac{4}{A / S}\right]
$$

- $K_{\text {Lin }}$ est le facteur d'environnement moyen.

\subsubsection{Appareillage de mesures}

Chaînes de mesures :
- Sonomètres BRUEL et KJAER

.. 2215 pour les mesures d'ambiance

.. 2209 pour les mesures aux points clés.

- Micros Bruel et Kjaer 4145

- Magnétophones NAGRA 4/2 et NAGRA 3

(ces matériels professionnels présentent l'avantage d'une très grande maniabilité lors des mesures sur site).

Matériel d'analyse :

- Analyseurs de fréquence : BRUEL et KJAER

- Programme de dépouillement: EDF/ACT sur HP 9845B

Divers :

- Source sonore de référence (SSR) : ventilateur $85 \mathrm{~dB}(\mathrm{~A})$

à $1 \mathrm{~m}$

- Pistolet d'alarme ARMINIUS HWIG $9 \mathrm{~mm}$

- Pistonphones BRUEL et KJAER 4220/30. 


\subsection{Connaissance des sources}

L'ensemble turbine-alternateur restant solidaire mécaniquement par l'intermédiaire de l'arbre, sans qu'il soit possible de "débrayer" l'une des parties rotatives par rapport aux autres, la séparation des sources nécessite deux hypothèses délicates :

- il n'y a pas de transmission "solidienne " au travers des dalles bétonnées séparant les divers étages:

- il n'y a pas de transmission aérienne par les conduits ouverts : puits de montage, escaliers de circulation, circuits de ventilation.

Dans le cas de Auzat, la connaissance de la transmission "solidienne" par mesures vibratoires n'a pas été acquise et nous n'avons pu avoir de preuve, même approximative, de la légitimité de cette première hypothèse. Il s'agit donc d'une pétition de principe, probablement inexacte dans certains cas (voir 1.2.1.3).

Concernant la transmission aérienne, la seule manière scientifique de l'éliminer aurait été d'utiliser la méthode intensimétrique.

En mesurant uniquement la pression acoustique selon la norme NF S 31 067, l'impact aérien des autres sources ne peut être apprécié qu'en considérant les différences entrainées par la mise en service des diverses isolations.

Sans entrer dans l'examen de détail des isolations qui sera fait au $\S 1.3$, on peut aller directement aux résultats utiles, à savoir :

- la transmission aérienne provoquée par la source alternateur (ALT) avant son cloisonnement était forte en direction de l'excitatrice (EXC, $8 \mathrm{~dB}(\mathrm{~A})$ ) et significative en direction des paliers turbine (TUR) et de l'aspirateur (ASP);

- une fois cette isolation lourde mise en place, les autres cloisonnements n'ont d'effet significatif qu'au voisinage immédiat de la source cloisonnée. Ceci s'applique en particulier à TUR et ASP. Quant à EXC, son isolation a un effet de 2 à $4 \mathrm{~dB}(\mathrm{~A})$ aux autres étages, probablement par suppression de la réflexion au plafond de la salle des machines:

- on peut donc légitimement conclure que la transmission aérienne d'une source à l'autre est négligeable durant la mesure des pressions acoustiques de chaque source, pour autant que l'isolation de l'alternateur soit en place, de même que, dans une moindre mesure, celle de l'excitatrice.

\subsubsection{Les sources principales}

1.2.1.1. L'alternateur constitue de loin la source principale, avec une puissance acoustique de 115,6 dB(A) (détails au $\S 1.5$ ). Les bandes de fréquence dominantes sont, dans l'ordre décroissant des amplitudes : $280-355 \mathrm{~Hz}$, $1120-1400 \mathrm{~Hz}, 90-110 \mathrm{~Hz}$ (figure 4). Elles mettent en jeu le nombre de pôles du ventilateur, le nombre de directrices et le nombre de pôles du rotor.

1.2.1.2. L'excitatrice représente la deuxième source importante, avec 102,6 dB (A).

La première remarque à faire est que la pression acoustique émise par le capotage latéral de EXC est de presque $10 \mathrm{~dB}$ inférieure à celle de sa partie supérieure ouverte (figure 5).
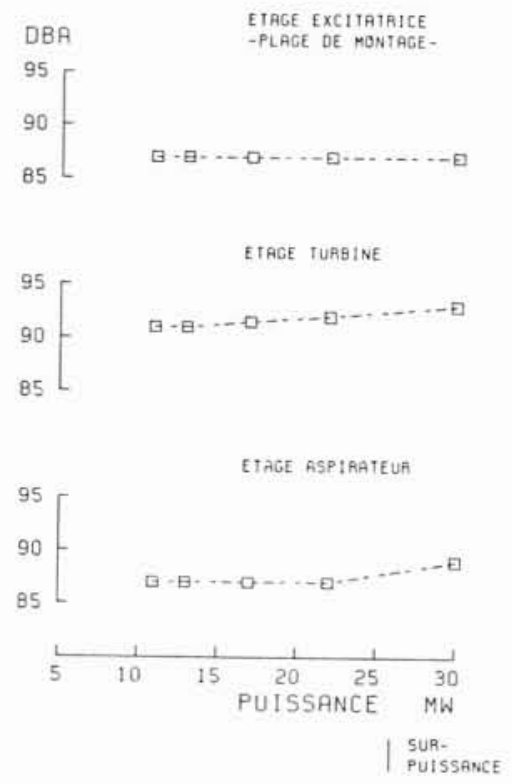

8. Influence de la charge sur les niveaux d'ambiance.

Le tableau 10 indique également une très forte réverbération dans la bande $63 \mathrm{~Hz}$, soit $13 \mathrm{~dB}$, qui provient essentiellement de la réflexion sur le plafond métallique de la salle des machines.

La deuxième remarque concerne le caractère peu différencié des spectres de pression; on ne trouve pas les pics bien marqués émis par ALT. Les niveaux maximaux restent ceux de la bande $280-355 \mathrm{~Hz}$.

1.2.1.3. Le "palier turbine" a une puissance de 98.6 $\mathrm{dB}(\mathrm{A})$, étant bien entendu qu'à cet étage sont reçus les bruits hydrodynamiques transmis par le flasque supérieur de la turbine.

Bien que moins différencié que celui de ALT, le spectre de pression de TUR (figure 6) manifeste une certaine similitude avec celui de ALT. En particulier, le pic $1120-1400 \mathrm{~Hz}$ est encore net alors qu'il correspond à une modulation entre passage des aubes du ventilateur et passage devant les directrices.

D'autre part, le pic maximal reste à $90-110 \mathrm{~Hz}$, qui correspond au bruit magnétique. Il semble donc se produire une transmission importante au travers du croisillon de l'alternateur.

1.2.1.4. La dernière source principale provient de l'aspirateur, avec 95,2 dB (A) de puissance émise : cette valeur est certainement surestimée par non-correction de la réverbération, l'exiguïté du local n'ayant pas permis de dégager un coefficient correctif $K$ vraisemblable.

Le spectre est assez plat (figure 7) avec un pic légèrement dominant à $56-90 \mathrm{~Hz}$ qui provient probablement du défilement des aubes devant les directrices, à la fréquence $\left(Z_{D}-Z_{R}\right) f_{0}$, soit $83 \mathrm{~Hz}$.

Mise à part la faible puissance acoustique émise, il faut remarquer que le régime de fonctionnement influe fort peu en valeur globale (A) sur la source ASP (figure 8). 
La bande des $63 \mathrm{~Hz}$ est la plus évolutive, avec $78 \mathrm{~dB}$ au régime optimal, $80 \mathrm{~dB}$ à charge partielle et $82 \mathrm{~dB}$ en surpuissance.

Ces faibles variations en fonction de la charge confirment le rôle dominant de la puissance acoustique de l'alternateur, rôle qui se verra confirmé par l'étude des isolations.

\subsubsection{Les sources secondaires}

Quatre pompes auxiliaires, soit d'eau, soit d'huile, constituent des sources secondaires dont la contribution a été contrôlée pendant l'arrêt des groupes hydro-électriques.

De manière sommaire mais concluante, on a mesuré le bruit à $1 \mathrm{~m}$ de chaque source.

Celles-ci étant de faibles puissances, la contribution du bruit de fond (usine proche, bruit d'eau dans le puits d'exhaure) ne peut être admise comme négligeable (jusqu'à $70 \mathrm{~dB}(\mathrm{~A}))$ par rapport aux résultats ci-dessous :

\begin{tabular}{|lccc|}
\hline Pompe & Vitesse & Puissance & $\begin{array}{c}\text { Niveau pression } \\
\text { sonore a i m } \\
\mathrm{dB}(\mathrm{A})\end{array}$ \\
\hline $\begin{array}{l}\text { Auxiliaire : } \\
\mathrm{tr} / \mathrm{min}\end{array}$ & $\mathrm{kW}$ & 76,5 \\
\hline $\begin{array}{l}\text { - pégulation } \\
\text { réfrigération }\end{array}$ & 1430 & 3 & 76 \\
- exhaure & 1425 & 8 & 77 \\
\hline
\end{tabular}

Ce niveau sonore étant inférieur de plus de $10 \mathrm{~dB}$ (A) à celui des sources principales, on peut considérer que la contribution des pompes auxiliaires au bruit général est nulle.

D'autres mesures en atelier sur ce type de matériel ont confirmé que la puissance acoustique des auxiliaires était négligeable, en tout cas pour des vitesses de rotation inférieures à $3000 \mathrm{tr} / \mathrm{mn}$. Rappelons à cette occasion que, selon les travaux de la commission spécialisée de la S.H.F., le niveau sonore dépend de la vitesse de rotation des pompes centrifuges selon l'expression :

$$
L_{P A}=37+10 \log \left(\frac{N . W}{n}\right) \pm 6 \mathrm{~dB}(\mathrm{~A})
$$

- $N$ en $\mathrm{tr} / \mathrm{mn}$

- $W$ en $\mathrm{kW}$

- $n$ nombre d'étages.

\subsection{Influence des isolations}

\subsubsection{Isolations lourdes}

Lors de la mise en route, un niveau sonore de $96 \mathrm{~dB}$ (A) s'établissait dans la salle des machines, obligeant le personnel à une protection auditive. Selon les premières investigations, les sources principales apparurent, à l'époque, être l'alternateur et l'aspirateur : des isolations furent mises en place à ces étages,
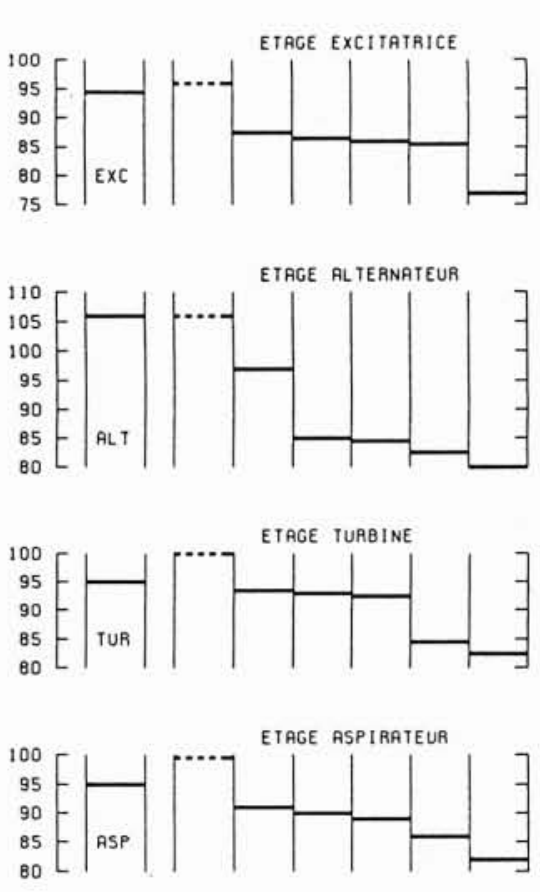

DBA

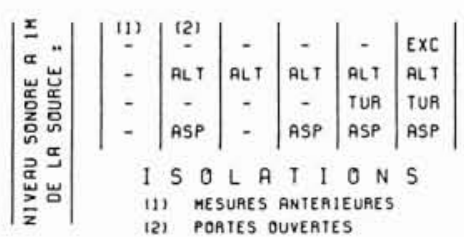

9. Mesures d'ambiance et influence des diverses isolations.

- autour des deux alternateurs à ventilation commune, cloison inamovible avec porte, d'épaisseur $100 \mathrm{~mm}$ composée de deux panneaux métalliques enserrant de la laine de roche. Atténuation intrinsèque : $40 \mathrm{~dB}(\mathrm{~A})$;

- devant la galerie du coude de l'aspirateur, cloison de même qualité avec porte. Cette cloison doit être retirée lors des démontages de la roue de turbine.

\subsubsection{Isolations légères}

Lors des mesures acoustiques de février 1983, deux isolations provisoires ont été mises en place :

- devant l'enceinte du palier turbine, une cloison Novopan de $22 \mathrm{~mm}$ vissée sur plaques caoutchouc, - autour de l'excitatrice, des panneaux de laine de verre d'épaisseur $75 \mathrm{~mm}$.

\subsubsection{Mesures comparatives}

La figure 9 donne les atténuations globales en $\mathrm{dB}(\mathrm{A})$ apportées à chaque étage lors de la mise en service progressive des isolations des sources principales.

- le cloisonnement avec porte fermée autour des alternateurs apporte une atténuation de :

$9 \mathrm{~dB}(\mathrm{~A})$ à l'étage excitatrice,

$20 \mathrm{~dB}(\mathrm{~A})$ à l'étage alternateur (extérieurs cloisons),

$7 \mathrm{~dB}(\mathrm{~A})$ à l'étage turbine,

$9 \mathrm{~dB}(\mathrm{~A})$ à l'étage aspirateur. 
- l'ouverture de la porte dans le cloisonnement alternateur provoque une majoration de $12 \mathrm{~dB}(\mathrm{~A})$ à l'étage alternateur,

pratiquement rien ailleurs.

- le cloisonnement aspirateur est d'une utilité quasi nulle, même à l'étage aspirateur.

- le cloisonnement (provisoire) de l'étage turbine apporte peu à l'étage excitatrice, mais :

moins $2 \mathrm{~dB}(\mathrm{~A})$ à l'étage alternateur,

moins $8 \mathrm{~dB}(\mathrm{~A})$ à l'étage turbine,

moins $3 \mathrm{~dB}(\mathrm{~A})$ à l'étage aspirateur.

(On rappelle que le bruit émis à l'étage turbine provient également de la source alternateur par transmission au travers du croisillon).

- l'isolation (provisoire) de l'excitatrice est très intéressante puisqu'elle apporte par elle-même :

moins $8 \mathrm{~dB}(\mathrm{~A})$ à l'étage excitatrice,

moins $3 \mathrm{~dB}(\mathrm{~A})$ à l'étage alternateur,

moins $2 \mathrm{~dB}$ (A) à l'étage turbine,

moins $4 \mathrm{~dB}(\mathrm{~A})$ à l'étage aspirateur.

- les niveaux absolus d'ambiance avec toutes isolations installées deviennent :

$77 \mathrm{~dB}(\mathrm{~A})$ à l'étage excitatrice salle machines,

$80 \mathrm{~dB}(\mathrm{~A})$ à l'étage alternateur,

$83 \mathrm{~dB}(\mathrm{~A})$ à l'étage turbine,

$82 \mathrm{~dB}(\mathrm{~A})$ à l'étage aspirateur.

\subsubsection{Conclusions concernant les isolations}

Les résultats précédents amènent à des conclusions assez fermes :

La puissance de chaque source doit être analysée, (même de manière sommaire) et non appréciée de manière subjective. En matière acoustique, le jugement sensoriel peut se révéler très inexact et la connaissance d'un niveau de pression sonore d'ambiance est en général insuffisante pour déterminer les origines du bruit mesuré. Lorsqu'il n'est pas possible de mettre en action séparément chacune des sources (solidarité des parties tournantes), et en attendant des méthodes d'investigation plus sophistiquées faisant appel notamment à l'intensimétrie, la démarche du §1.3.3. permet de les caractériser et de définir un plan cohérent d'isolation.

- la méthode la plus efficiente pour réduire du bruit dans toutes les zones de circulation de la centrale consiste à isoler toutes les sources sonores dont la puissance contribue de façon significative au bruit ambiant, même avec des cloisons ou capotages légers.

En revanche, pratiquer des isolations lourdes sur certaines sources et aucune isolation sur d'autres, conduit à un résultat peu satisfaisant.

L'exemple des deux isolations provisoires de l'excitatrice et du palier turbine est très éclairant, puisque chacune, à son étage, a apporté plus d'atténuation que ne le fait l'isolation lourde de l'alternateur;

- dans la mesure du possible, on doit éviter de cloisonner en bloc deux sources appartenant à deux groupes différents, car la protection du personnel en cas d'entretien sur un seul groupe devient impossible;

- même dans une centrale dont les parties tournantes sont aussi bruyantes et le génie civil aussi réverbérant qu'à Auzat, la combinaison des diverses isolations permet de parvenir à des niveaux d'ambiance sonore largement acceptables à tous les étages et on peut constater l'atténuation totale obtenue par le tableau suivant :

\begin{tabular}{|l|c|c|}
\hline & $\begin{array}{c}\text { Avant } \\
\text { toute } \\
\text { isolation } \\
\mathrm{dB}(\mathrm{A})\end{array}$ & $\begin{array}{c}\text { Apres } \\
\text { toutes } \\
\text { isolations } \\
\mathrm{dB}(\mathrm{A})\end{array}$ \\
\hline $\begin{array}{l}\text { Salle machines } \\
\text { Etage : }\end{array}$ & 96 & 77 \\
$\begin{array}{l}\text {-alternateur } \\
\text { turbine }\end{array}$ & 106 & 80 \\
\hline aspirateur & 99 & 83 \\
\hline
\end{tabular}

\subsection{Correction de réverbération}

\subsubsection{Application de la norme NFS 31067}

Le tableau 10 résume les résultats pour chacune des sources comme indiqué au $\S 1.1 .2$.

$\mathrm{Si}$ les valeurs obtenues pour EXC et ALT peuvent être crédibles (moyennes respectives 4,3 et $5,1 \mathrm{~dB}$ ), en revanche, celles des enceintes turbine et aspirateur ne sont pas exploitables (valeurs négatives).

Lorsque l'application de la norme conduit finalement à réduire la surface de mesurage à un seul plan (porte d'accès d'un local très confiné), toute déduction de la correction de réverbération par cette méthode paraît illusoire.

\subsubsection{Utilisation des temps de réverbération}

Le tableau 11 (page suiv.) donne les $T$, obtenus aux emplacements des sources et les coefficients $K_{L i n}$ moyens que nous en avons déduits.

$\mathrm{Si}$ nous comparons ces valeurs à celles obtenues selon la norme, il apparaît que la correction de réverbération ne peut qu'être forte aux étages turbine et aspirateur, ce qui est logique compte tenu du confinement.

\begin{tabular}{|c|c|c|c|c|c|c|c|c|c|c|}
\hline $\begin{array}{l}\text { OCTAVE } \\
(\mathrm{Hz})\end{array}$ & & 31,51 & 63 & 125 & 250 & 500 & 1000 & 2000 & 4000 & 8000 \\
\hline $\begin{array}{l}\text { LW SSR } \\
\text { étalonnage }\end{array}$ & & & 82,2 । & 85,4 । & 87,1 | & $87,7 \mid$ & 90,4 & $|87,8|$ & 89,5 & $\mid 88,8$ \\
\hline LW SSR & IEXC & 93,11 & 95,4 & 187,7 & $|89,3|$ & $\mid 93,0$ & 197,5 & 192,8 & 191,8 & 186,0 \\
\hline \multirow{3}{*}{$\begin{array}{l}\text { niveaux } \\
\text { bruts } \\
\text { relevés } \\
\text { sur site }\end{array}$} & IALT & 94,9 & 92,1 & 187,9 & $|90,5|$ & 91,3 & 394,8 & 93,0 & 192,7 & 87,3 \\
\hline & ITUR & 85,4 & 77,6 & 82,2 & $|82,2|$ & 85,6 & $5 i 88,6$ & 185,9 & 185,6 & 79,9 \\
\hline & ASP & 89,8 & 84,0 & i9,9 & $|80,1|$ & $i^{83,5}$ & $\varliminf^{i}$ & 86,6 & 187,6 & 83,3 \\
\hline \multirow{4}{*}{$\begin{array}{l}\quad \mathrm{K} \\
\text { facteur } \\
\text { de } \\
\text { correction } \\
\text { d'environ- } \\
\text { nement }\end{array}$} & IEXC & 3,11 & 13,2 & 12,3 & $|2,2|$ & 5,3 & $3 \mid 7,1$ & 15,0 & I 2,3 & $?$ \\
\hline & IALT & 4,9 & 9,9 & 2,5 & 3,4 & 3,6 & 4,4 & 5,2 & 3,2 & $?$ \\
\hline & ITUR & $?$ & $?$ & $?$ & $?$ & $?$ & $?$ & ? & $?$ & $?$ \\
\hline & iASP & ? & 1,8 & ? & i ? & ? ? & $?$ & ? & ? & $?$ \\
\hline
\end{tabular}

10. Correction de la réverbération selon la norme NFS 31067 


\begin{tabular}{|l|c|c|c|c|c|}
\hline & & EXC & ALT & TUR & ASP \\
\hline$T_{r}$ & $\mathrm{~S}$ & 4,5 & 2,6 & 4,9 & 4,5 \\
$V$ & $\mathrm{~m} 3$ & 3130 & 287 & 50,6 & 27,4 \\
$\mathrm{~S}$ & $\mathrm{~m} 2$ & 68,4 & 30,2 & 1,7 & 1,3 \\
$\mathrm{~A} / \mathrm{S}$ & - & 1,6 & 0,6 & 0,97 & 0,75 \\
$\mathrm{~K}_{\mathrm{L} \text { in }}$ & $\mathrm{dB}$ & 5,4 & 8,9 & 7 & 8 \\
\hline
\end{tabular}

11. Estimation de la correction d'environnement d'après le temps de réverbération.

\subsection{Résultats finals selon la norme NFS 31067}

\subsubsection{Niveaux de pressions surfaciques et de puis- sances d'émission en pondération $A$}

Les pressions acoustiques surfaciques à $1 \mathrm{~m}$ des sources et les puissances acoustiques de celles-ci sont résumées ci-dessous :

\begin{tabular}{|l|r|r|r|r|}
\hline & EXC & ALI & TUR & ASP \\
\hline TPAf $(\mathrm{dB}(A))$ & 84,2 & 101 & $96,3 * 1$ & $94,1 *$ \\
\hline $\mathrm{S}\left(\mathrm{m}^{2}\right)$ & 68,4 & 30,2 & 1,7 & 1,3 \\
\hline$W_{A}(\mathrm{~dB}(\mathrm{~A}))$ & 102,6 & 115,6 & $98,6 * 1$ & $95,2 *$ \\
\hline
\end{tabular}

- (les valeurs pour TUR et ASP ne sont pas corrigées de la réverbération, car les valeurs de $K$ ne sont pas crédibles)

On constate la prédominance des puissances acoustiques émises par les parties tournantes électriques, prédominance qui serait encore accentuée si la correction de réverbération pouvait être appliquée aux étages turbine et aspirateur.

\subsubsection{Courbe d'évaluation C 80 DE ISO}

Une autre possibilité d'évaluation des niveaux de pression acoustiques consiste à situer les spectres obtenus par bandes d'octaves relativement aux courbes d'évaluation ISO.

Le graphique $n^{\circ} 12$ montre que EXC ne dépasse pas l'indice $\mathrm{C} 80$ alors que ALT atteint C 100 (après correction d'environnement).

Concernant TUR et ASP, nous ne pouvons parvenir à la même comparaison avec les indices ISO, compte tenu des coefficients d'environnement $K$ inexploitables. Néanmoins, l'examen des temps de réverbération nous indique un ordre de grandeur de cette réverbération allant de 7 à $8 \mathrm{~dB}$. Les niveaux sonores TUR et ASP corrigés ne dépassent donc pas l'indice C 85 .

\subsection{En résumé}

1.6.1. Le régime de fonctionnement des machines influe fort peu sur le niveau de bruit aux différents étages de la centrale. La puissance sonore émise est donc liée pour une part très prépondérante à la vitesse de rotation, qui est constante, et pour une part marginale, au débit variable traversant la turbine.
1.6.2. Les puissances sonores de chaque source principale décroissent dans l'ordre suivant :

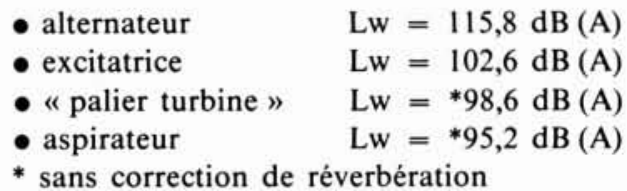

1.6.3. Les puissances sonores des pompes auxiliaires apparaissent comme négligeables, vis-à-vis des autres sources non insonorisées.

1.6.4. La méthode la plus efficace de réduction du bruit consiste à isoler toutes les sources importantes, même sommairement. En revanche, l'isolation lourde de certaines sources et l'absence d'isolation sur d'autres conduit à un résultat décevant. D'autre part, cloisonner deux sources dans une même enceinte provoque une difficulté lors de l'entretien d'une source.

1.6.5. En cloisonnant, même sommairement les quatre sources principales, les niveaux d'ambiance obtenus à chaque étage sont largement acceptables puisqu'ils vont de $77 \mathrm{~dB}(\mathrm{~A})$ à $83 \mathrm{~dB}(\mathrm{~A})$.

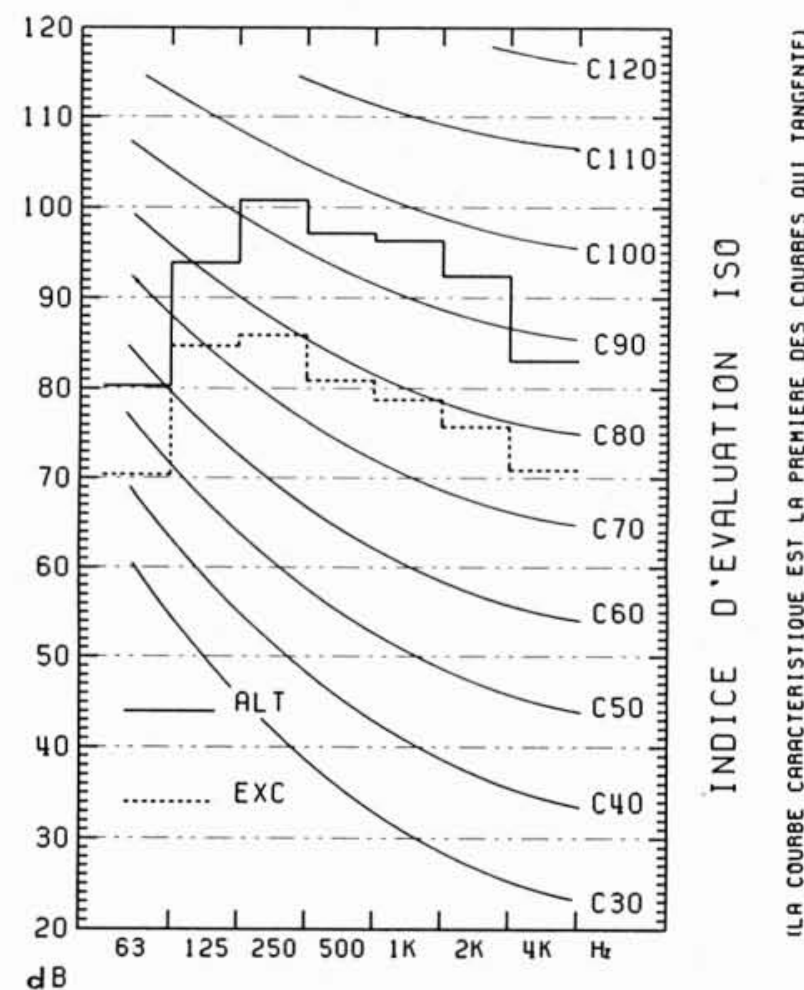

BANDES DE FREQUENCES EN OCTAVES

12. Comparaison aux indices d'évaluation ISO des niveaux de pression acoustique surfacique de l'alternateur et de l'excitatrice. 


\section{Une démarche prospective validée}

Electricité de France utilise depuis longtemps une méthode acoustique prévisionnelle pour concevoir ses installations de production thermique, au regard de la protection de l'environnement extérieur aux usines. Des règles d'établissement des cahiers des charges ont également été fixées pour la limitation des niveaux sonores intérieurs aux usines de production nucléaire.

Après l'expérience d'Auzat, la décision fut prise d'entreprendre une démarche analogue pour les usines hydrauliques afin d'obtenir, dès la mise en service des usines, le respect des niveaux sonores admissibles.

L'action s'est déroulée en trois phases.

\subsection{Définition des objectifs}

L'étude des textes officiels (normes françaises ou étrangères), des recommandations internes à l'Electricité de France et l'évolution probable à long terme de la législation nationale ou européenne conduisent à préconiser deux valeurs de base. Ces valeurs correspondent aux deux situations auxquelles le personnel d'exploitation est confronté :

- lorsque l'installation est en service : l'objectif à respecter est de $90 \mathrm{~dB}$ (A) sans protection auditive sur l'ensemble des planchers dont l'accès permanent est nécessité par l'exploitation.

- lorsqu'un groupe est à l'arrêt, ou en entretien, l'objectif assigné est de $80 \mathrm{~dB}(\mathrm{~A})$ : cet objectif doit être respecté sur l'ensemble des zones où s'effectuent les opérations d'entretien.

Dans cette approche, et compte tenu de l'état actuel de la technique, les niveaux sonores ne sont pas limités sur les planchers de service non accessibles en permanence : leur accès implique alors le port d'une protection auditive.

Pour tous les autres locaux tels que locaux d'exploitation et salles de commande, EDF se fixe un objectif de $60 \mathrm{~dB}(\mathrm{~A})$.

Cette approche a reçu l'accord des Services d'exploitation et des Responsables de la Sécurité : l'expérience montre également que le personnel d'exploitation accepte volontiers la contrainte de la protection individuelle dans certaines zones, si dans le reste de l'usine, les niveaux sonores sont suffisamment faibles.

\subsection{Connaissance du problème}

Cette partie de l'étude, dont l'expérience d'Auzat illustre l'un des aspects, s'est déroulée sur trois années, en menant de manière méthodique, une série d'investigations sur plus de dix unités en exploitation, investigations menées en collaboration avec le Service de la Production hydraulique et la Direction de l'Equipement.

Ces analyses ont porté notamment sur les centrales de : Auzat, Montézic, Nentilla, Le Baous, Tolla-Occana, Pouget, Sisteron, Vignotan, Brassac, La Croux, Neppes, Saint-Etienne-Cantalès, Motz, la Jourdanie et Révin.

Les niveaux sonores imputables aux divers éléments constitutifs ont pu ainsi être mieux connus, de même que l'efficacité de certaines dispositions techniques. Il s'est confirmé le grand nombre de situations qui peuvent être rencontrées suivant le type de chute équipée, l'époque de mise en service et le savoir-faire du concepteur.

Cette enquête a montré, en particulier, que le respect de niveaux sonores acceptables pour le personnel d'entretien ne pouvait raisonnablement être atteint que par l'indépendance acoustique des divers groupes d'une même usine.

Cette disposition, tout à fait praticable dans le cas des groupes verticaux, présente toutefois des difficultés d'application dans le cas de groupes horizontaux, pour des raisons de manutention.

A l'issue de cette phase d'analyse, il est devenu possible de mener des études prévisionnelles sur plan pour plusieurs centrales en cours de conception ou de sur-équipement. Les dispositions conseillées ont été définies en associant aux concepteurs, un spécialiste des questions acoustiques de manière à assurer l'efficacité et la faisabilité au moindre coût de ces dispositions.

\subsection{Elaboration d'un guide-type}

Les dispositions à prendre sont de trois types :

- Désolidarisation des machines et de leurs massifs supports vis-à-vis des bâtiments d'une part, désolidarisation des bâtiments usine vis-à-vis des locaux d'exploitation d'autre part;

- Traitement absorbant de la salle des machines, réalisé de préférence dans la phase même de coffrage par la technique du coffrage perdu, comportant une face acoustiquement absorbante. Le coût du traitement est alors très réduit.

- Mise en place de portes acoustiques dans les espaces intergroupes, les accès aux divers étages et les cuvelages des machines.

Ces dispositions intègrent les problèmes de ventilation et les orifices laissés libres à cet effet sont munis de silencieux dont l'atténuation est homogène à celle des portes ou cloisons.

L'application de ces recommandations dont l'ensemble est présenté dans un "GUIDE TYPE " à la destination du concepteur et du bureau d'étude, n'entraîne pas de dépenses excessives. Cependant, il est indispensable que leur mise en œuvre soit prise en compte suffisamment tôt, faute de quoi elles s'avèreraient moins efficaces ou inapplicables : en effet, le découplage mécanique des locaux par exemple, doit être inclus dans le processus même de leur réalisation, sinon il devient irréalisable - ou d'un prix de revient prohibitif s'il conduit à démolir, en cours de chantier, tout ou partie du gros-œuvre.

Ces principes ont été mis en œuvre à titre de dèmonstration et de validation pour deux usines.

- l'usine de Montézic;

- l'usine de Saint-Guillerme.

\subsection{L'expérience de Montézic}

L'usine de Montézic est équipée de quatre groupes à axe vertical comportant chacun un alternateur-moteur de 

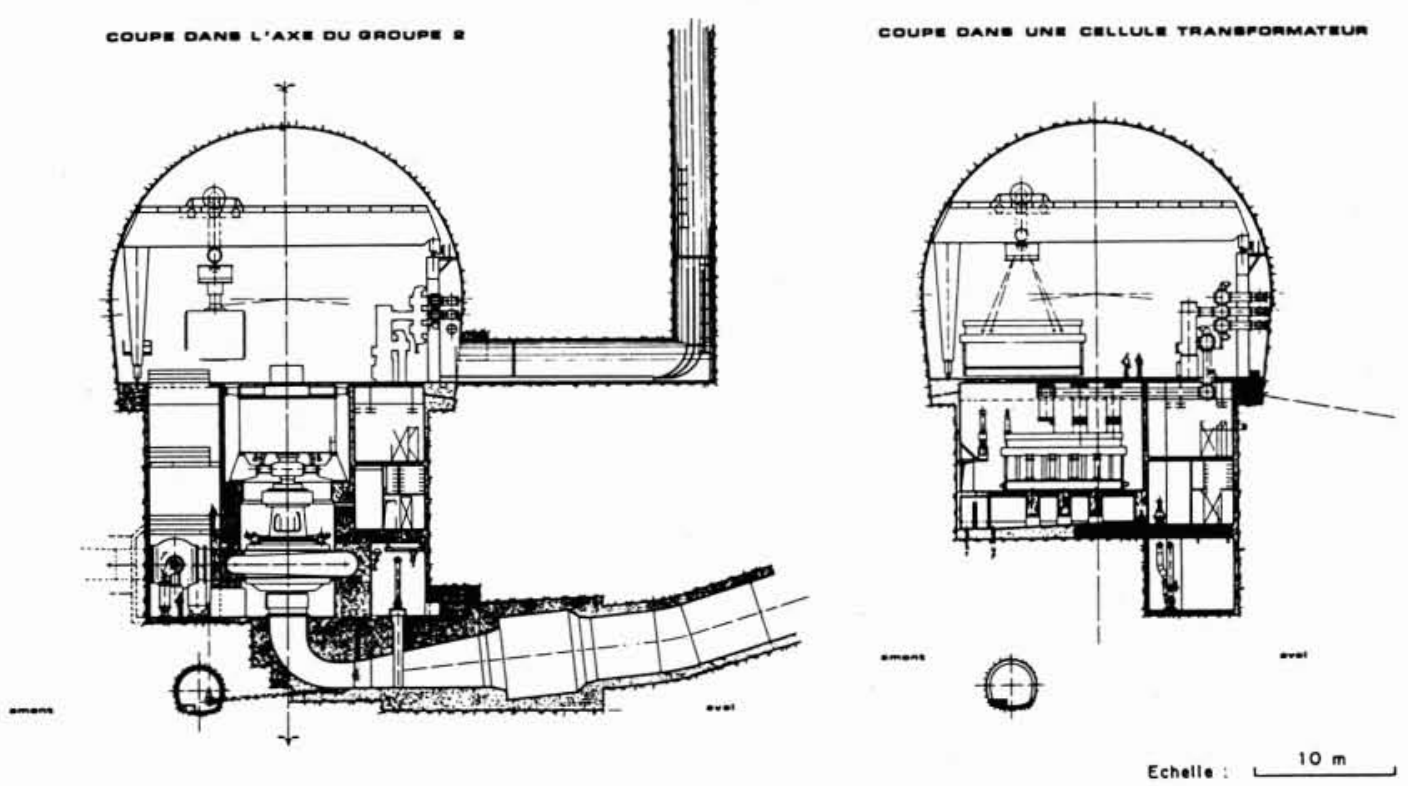

13. Centrale de Montézic - Coupes.

SALLE DES MACHINES

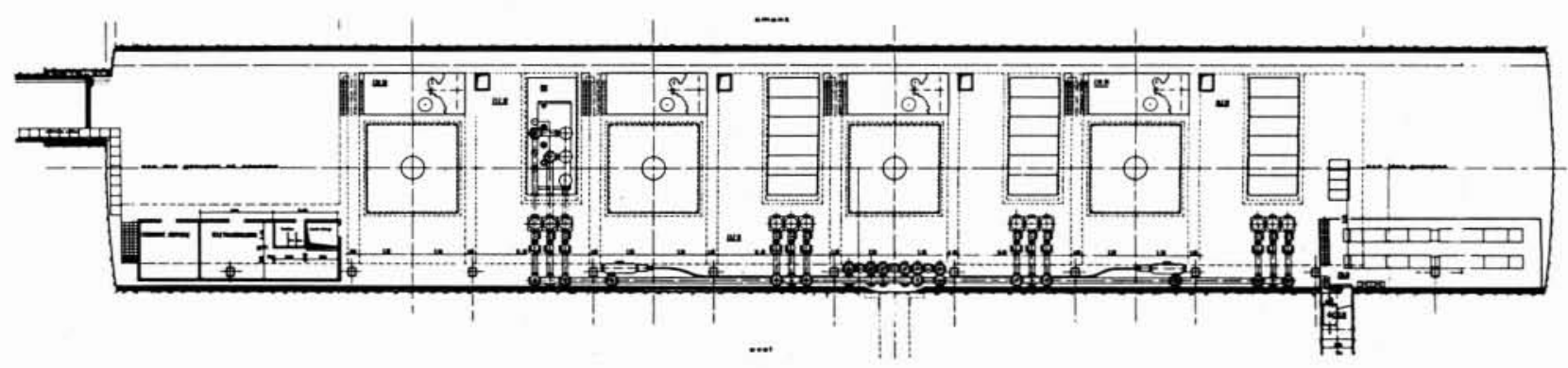

ETAGE ELECTRIQUE

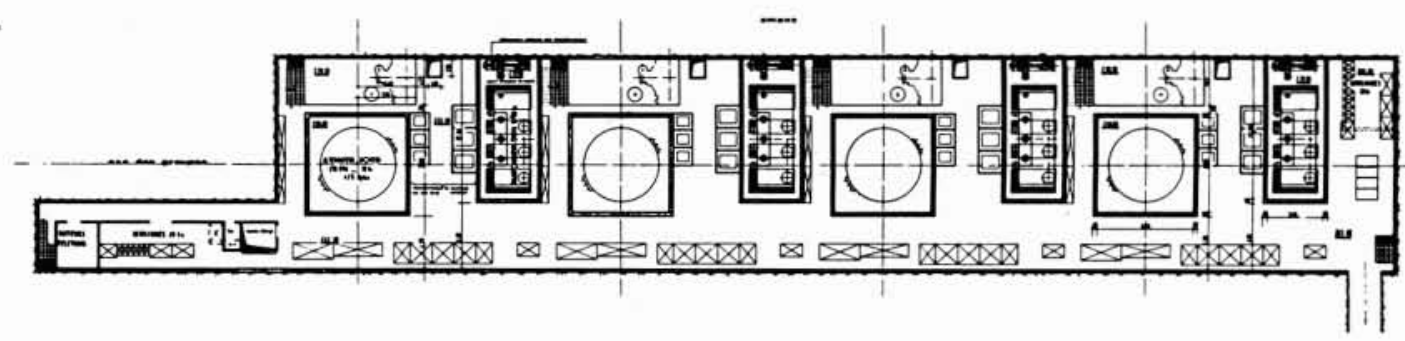

14. Centrale de Montézic - Vues en plan.

Echelle: $10 \mathrm{~m}$ 
ETAGE EXPLOITATION

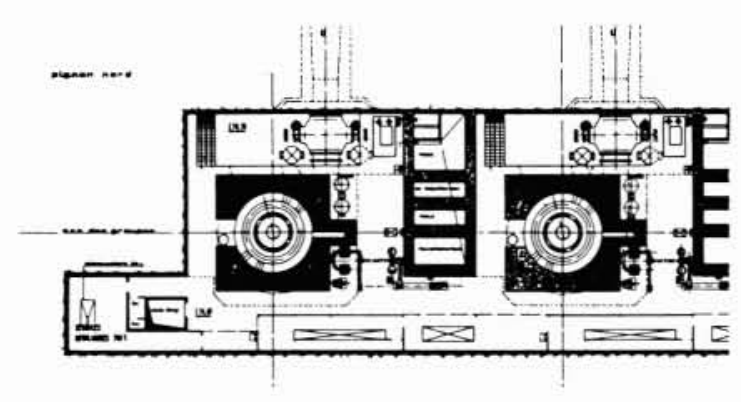

15.

. Centrale de Montézic - Vues en plan.
ETAGE HYDRAULIQUE

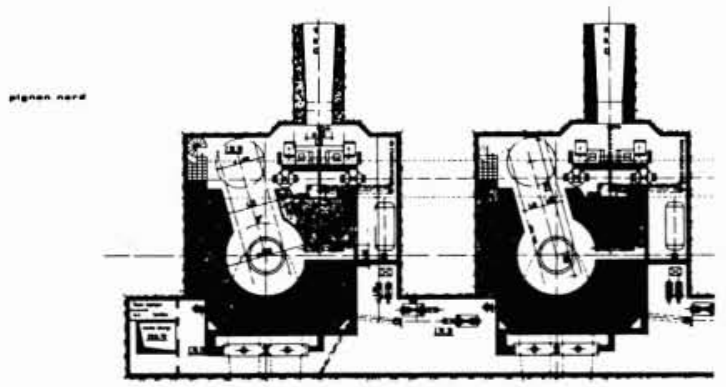

$10 \mathrm{~m}$
$250 \mathrm{MW}$ tournant à $428 \mathrm{tr} / \mathrm{mn}$ et d'une pompe turbine réversible de type Francis.

Ces matériels sont installés dans l'excavation constituant l'usine qui comporte en outre les robinets sphériques, les transformateurs auxiliaires, les transformateurs principaux, les tableaux et armoires de contrôle commande, le poste auxiliaire $20 \mathrm{kV}$, le poste $400 \mathrm{kV}$ et le convertisseur statique de démarrage. Les figures 13 à 15 donnent une coupe de l'usine et une vue en plan des différents étages.

L'analyse acoustique de cet équipement selon les principes énoncés ci-avant, n'a pu être entreprise qu'en fin de réalisation et la première campagne de mesures de contrôle a été menée alors que le premier groupe était déjà en service. Les aménagements suivants ont alors été effectués :

- en salle de machines (niveau 253,30) :

- désolidarisation des locaux d'exploitation par rapport au plancher et mise en place de portes acoustiques,

- fermeture par dalles des fosses des transformateurs principaux, avec mise en place d'un joint d'étanchéité amortisseur de vibrations.

- traitement des platelages alternateurs par mise en place d'un tapis néoprène chargé au plomb et d'une dalle antidérapante en néoprène :

- à l'étage "électrique " (niveau 241,40) :

- mise en place de portes acoustiques sur l'accès à la cellule transformateur, l'accès au cuvelage alternateur, et le couloir d'accès au puits de sortie de roue.

- à l'étage hydraulique $(236,60)$ :

- mise en place de portes acoustiques sur les cuvelages turbine,

- réalisation de cabines insonorisées autour des armoires de contrôle commande,

- confection d'une paroi séparative entre groupes;

- traitement par calorifuge acoustique des by-pass.

- à l'étage hydraulique (niveau 230,50) :

- porte acoustique de type guillotine sur l'orifice de sortie de roue,
- porte acoustique sur l'accès de l'aspirateur,

- porte acoustique et silencieux sur le local compresseur, - porte acoustique sur l'accès à la station d'exhaure.

Chacun des équipements d'isolation (portes et cabines) aucune atténuation de l'ordre de $30 \mathrm{~dB}$ (A) pour le spectre moyen des matériels bruyants.

Malgré la prise en compte un peu tardive des problèmes d'isolation acoustique, les résultats obtenus sont proches des objectifs fixés. Quelques dépassements subsistent en certains points et pour certaines conditions de fonctionnement. On estime que sans ces aménagements les ambiances sonores auraient été de 10 à $15 \mathrm{~dB}$ (A) supérieures aux valeurs obtenues actuellement à savoir : 90 $\mathrm{dB}(\mathrm{A})$ pour la plage de fonctionnement 0 à $210 \mathrm{MW}$ et à $94 \mathrm{~dB}(\mathrm{~A})$ pour la plage 220 à $250 \mathrm{MW}, 80 \mathrm{~dB}(\mathrm{~A})$ pendant les opérations d'entretien en salle des machines (plancher supérieur des excitatrices) et étage d'exploitation (plancher d'accès au palier turbine), $87 \mathrm{~dB}(\mathrm{~A})$ pour les étages " électrique " et "hydraulique ".

Les exploitants considèrent que les équipements antibruit n'apportent pas de gêne en exploitation normale ou pour l'entretien de l'usine à l'exception d'une porte à l'étage sortie de roue, porte qu'il faudra déposer lors de la manutention de pièces importantes.

\subsection{L'expérience de Saint-Guillerme}

Pour cette usine souterraine gravitaire qui comporte deux groupes Francis verticaux d'une puissance unitaire de 58 MW (figure 16), les prescriptions acoustiques du guide type ont été prises en compte dès le stade de l'avant-projet.

Les objectifs recherchés ont été largement atteints puisque, en salle des machines, le niveau sonore n'excède en aucun point la valeur de $85 \mathrm{~dB}(\mathrm{~A})$ pour le fonctionnement à puissance nominale et $79 \mathrm{~dB}(\mathrm{~A})$ sur l'ensemble d'un groupe à l'arrêt pour entretien, l'autre étant à pleine puissance.

On notera que sur la plage de démontage de l'un des groupes, l'autre étant à l'arrêt, le niveau sonore n'est que de $72 \mathrm{~dB}(\mathrm{~A})$. L'usine de Saint-Guillerme est ainsi l'une 


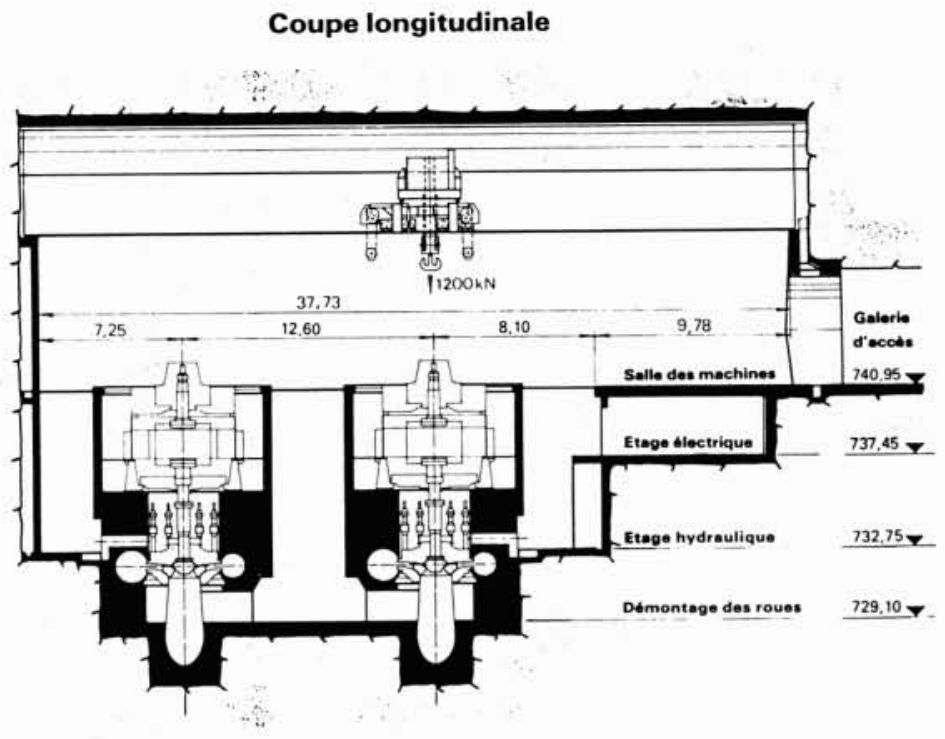

16. Centrale de St-Guillerme.

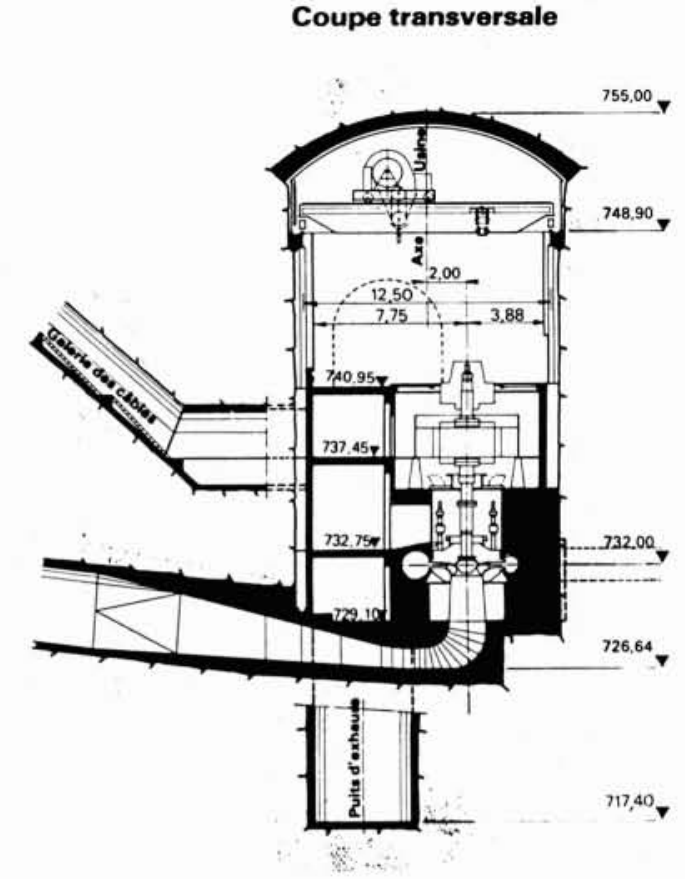

des plus silencieuse de l'ensemble du parc hydraulique d'Electricité de France.

Pourtant ce résultat a été obtenu pour un investissement limité à $0,4 \%$ du coût de l'usine, et sans allongement de la durée du chantier, grâce à deux éléments convergents :

- la prise en compte du problème dès la conception de l'usine;

- le contrôle continu de la réalisation des dispositions d'isolation acoustique.

Les deux expériences présentées ci-dessus confirment le bien-fondé de la démarche et l'efficacité des dispositions préconisées dans le guide est d'ailleurs un outil évolutif qui s'adaptera aux nouvelles possibilités techniques et aux matériels futurs.

\section{Quelques orientations pour l'avenir}

Au-delà de la démarche présente, il devient possible d'aller plus loin encore - si cela s'avère utile - dans la prévision des niveaux sonores intérieurs ou dans l'estimation des puissances sonores rayonnées par les matériels et la définition des améliorations à apporter.

\subsection{Acoustique prévisionnelle interne}

Le problème se pose en termes simples et généraux : connaissant la géométrie d'un local et les caractéristiques acoustiques de ses parois, peut-on calculer les niveaux sonores en tout point de ce local, pour une disposition connue de sources sonores (machines) placées dans ce local - sources sonores dont on connait la puissance rayonnante en fonction de la fréquence ?

Pour le physicien, ce problème peut être abordé suivant deux approches:

- les cas où le son est émis à une ou quelques fréquences bien déterminées - et stables - le plus souvent relativement basses dans le domaine auditif de l'homme (50 à 100 $\mathrm{Hz}$ par exemple). Il apparaît alors des phénomènes ondulatoires que l'on perçoit sous forme d'ondes stationnaires ou d'interférences. Ces cas doivent être étudiés fréquence par fréquence. Ils relèvent de l'équation de Helmoltz :

$$
p \frac{1}{c^{2}} \frac{\partial^{2} p}{\partial t^{2}}=S(t)
$$

Le rapport de la longueur d'onde des sons rayonnés $(\lambda=c / f)$ aux dimensions du local (et des machines) détermine l'allure du champ résultant.

- les cas où l'on peut considérer que l'énergie sonore rayonnée par les sources (machines) placées dans le local se propage comme la lumière, perdant à chaque réflexion sur les parois du local, une fraction de l'énergie du " rayon sonore ". Cette approche est utilisée dans le domaine des fréquences moyennes et aiguës et cette approximation hardie - ou toute notion ondulatoire a disparu - s'avère tout à fait efficace et d'une étonnante précision : ainsi, dès lors qu'un grand nombre de fréquences est à prendre en compte, la résolution de l'équation de Helmoltz qui devient rapidement impraticable peut-être remplacée par une méthode beaucoup plus simple et plus rapide, tout à 
fait utilisable avec un ordinateur de puissance et de capacité mémoire modestes. Différents codes de calcul ont été élaborés selon ces deux approches et les comparaisons calcul-expérience ont permis de vérifier la validité de ces outils.

La figure 17 donne par exemple les résultats des mesures (A) et calculs effectués (B) lors de l'étude acoustique de la centrale d'Eygliers, mise en service en mai 1982.

La figure $A$ est la carte des niveaux sonores observés dans la salle des machines avec deux groupes (10,4 MW) en service. Le niveau le plus faible est de $94,7 \mathrm{~dB}(\mathrm{~A})$ et le niveau le plus élevé de $99,8 \mathrm{~dB}$ (A).

La figure $B$ donne la situation qui existerait avec un capotage des turbines ayant une atténuation de $15 \mathrm{~dB}(\mathrm{~A})$ : le niveau minimum est passé à $90,5 \mathrm{~dB}(\mathrm{~A})$ et le niveau maximum est resté à $98,5 \mathrm{~dB}$ (A) (il est dû à la ventilation de l'alternateur).

Ce type de calcul est maintenant couramment appliqué à toutes les installations futures d'Electricité de France, ou aux installations qui sont à rénover : on peut ainsi tester avec une grande finesse différentes hypothèses d'implantation de sources sonores ou de traitement acoustique des locaux pour optimiser la solution à retenir, suivant certains critères : coût de l'insonorisation, confort acoustique dans certaines zones - et même compréhension des messages sonores transmis dans ces locaux.

On retiendra que la précision des calculs - en terme de niveaux sonores - peut atteindre le décibel c'est-à-dire qu'elle est du même ordre de grandeur que le seuil de sensibilité de l'oreille à une variation de niveau de bruit.

Bien entendu, ces calculs supposent que la puissance rayonnée sous forme de bruit par les différents matériels placés dans l'usine soit connue. Les acousticiens ont développé plusieurs méthodes pour caractériser les machines en tant que sources sonores (Normes NFS 30-006/31-022 à 027/31067-68). L'acoustique prévisionnelle permet de donner une réponse nouvelle à cette question comme on le verra ci-après.

\subsection{La détermination de la puissance acoustique des sources}

Il s'agit du problème inverse du précédent : connaissant la géométrie d'un local et les caractéristiques acoustiques de ses parois, peut-on calculer la puissance acoustique rayonnée par les machines en service, dès lors que l'on dispose des niveaux sonores en un certain nombre de points du local?

Sur le plan du principe, le problème n'a pas une solution unique : on démontre aisément que plusieurs combinaisons de sources de puissances sonores adéquates peuvent conduire à la même carte de bruit.

Cependant, si l'on suppose connue la position et la directivité des sources et notamment des plus puissantes d'entre elles, le calcul de la puissance de chacune d'elles est possible et le problème n'a qu'une solution.

Les méthodes d'identification développées par Electricité de France procèdent d'une idée simple : à partir d'un jeu de puissances assignées aux sources - dont les positions sont connues - on peut calculer la " carte de bruit ». Il suffit alors d'utiliser un algorithme itératif pour
Fig. A

SITUATION ACTUELLE - NON INSONORISEE - 2 GROUPES EN SERVICE - OB (A)
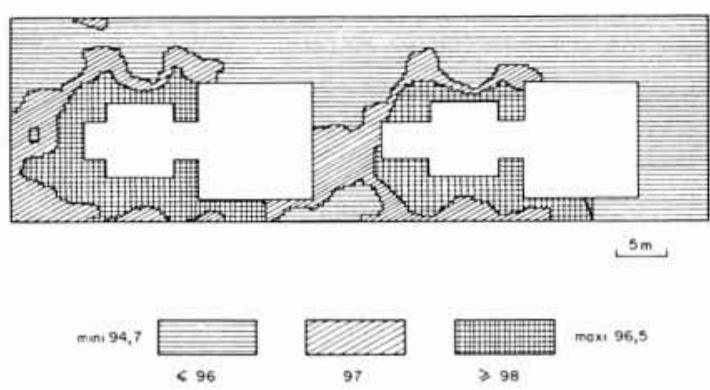

Fig $B$

2 GROUPES EN SERVICE - TURBINE INSONORISEE - [15 dB(A)]
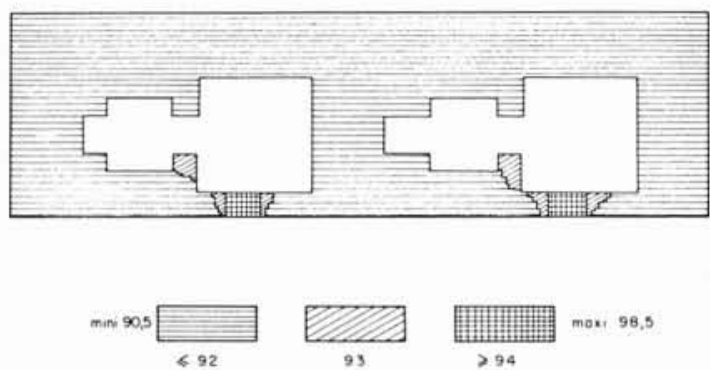

17. Centrale d'Eygliers: courbes de niveau sonore A) mesurées B) calculées.

caler au mieux ces puissances, de telle sorte que l'écart entre les cartes du bruit calculé d'une part, et mesuré d'autre part, soit minimal.

Il faut d'abord disposer d'une bonne représentation de la carte de bruit réelle dans le local étudié.

Il faut ensuite disposer d'un modèle de calcul des niveaux sonores: actuellement, les modèles utilisés sont de type "énergétique " et ne peuvent donc prendre en compte les situations particulières : interférences et ondes stationnaires signalées précédemment.

Pour les cas simples de salle de petites dimensions où rayonnent des sources physiquement séparables, on peut utiliser un modèle de calcul où le niveau sonore en un point est représenté par la superposition d'un champ direct et d'un champ réverbéré supposé uniforme.

La figure 18 donne la carte des différences de niveaux calculées et mesurées dans le cas d'une validation en salle semi-réverbérante : l'écart maximal est de $\pm 1 \mathrm{~dB}$.

Ce modèle a été utilisé pour reconstituer les niveaux sonores antérieurs à l'année 1977, dans une centrale hydraulique (Monistrol d'Allier) où des travaux importants avaient amené une réduction du nombre des groupes en service : partant de la carte actuelle (1984) dans les conditions d'exploitation présentes, on a " reconstruit " la carte existant avant la modification de cet aménagement.

Dans le cas général, on utilise la méthode des rayons décrite ci-avant : le champ réverbéré étant variable suivant la zone de la salle. Ce modèle est bien adapté aux salles de grandes dimensions et/ou avec un grand nombre de sources. 

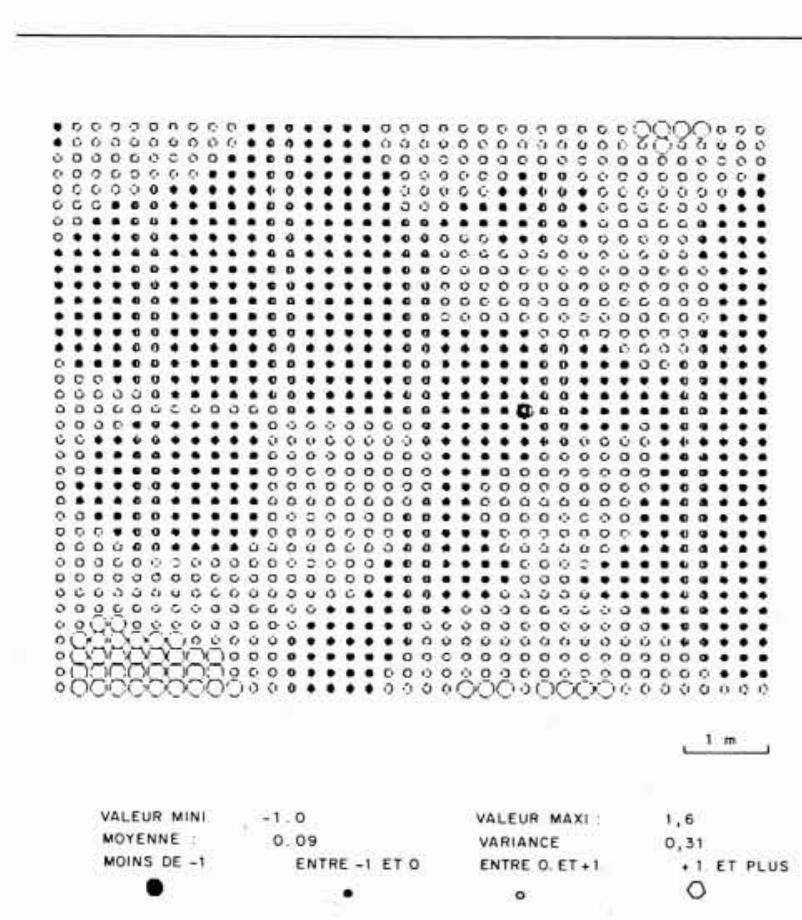

18. Carte des différences de niveaux sonores calculés et mesurés en salle semi-réverbérante.
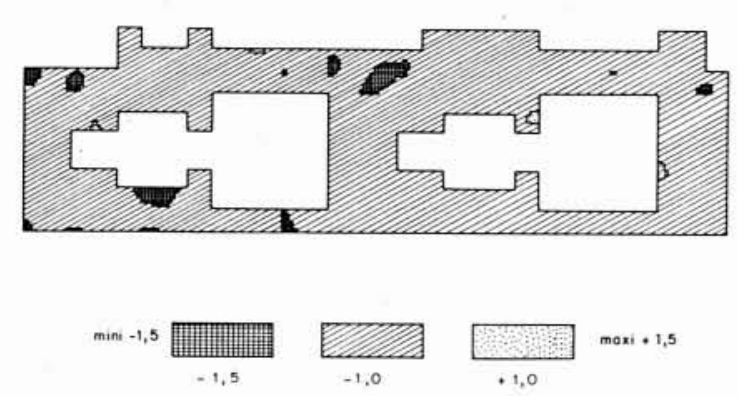

19. Centrale d'Eygliers - Calcul avec le code RAYON. Ecarts entre mesure et calcul.

La figure 19 donne pour la salle des machines de la centrale d'Eyglier, l'écart entre mesure et calcul des niveaux sonores en valeur globale A pour une configuration de 14 sources sonores représentant un turbo alternateur de 10,4 MW (turbine Francis double).

La méthode utilisée permet une identification des sources dont la fréquence est comprise entre deux valeurs : celle de la source la plus puissante, en limite supérieure, et en limite inférieure, une valeur de 15 à $20 \mathrm{~dB}$ plus faible.

Bien que cette méthode nécessite encore quelques approfondissements, elle constitue déjà un outil très performant: à partir d'une simple carte de bruit suffisamment précise - et de la connaissance des caractéristiques du local, on peut connaitre les sources qu'il faudra nécessairement traiter pour obtenir des réductions de niveaux ambiants allant jusqu'à $15 \mathrm{~dB}$ et parfois davantage, suivant les types de source et de locaux.

\section{Conclusion}

La réalisation d'équipements hydrauliques silencieux, qui résultait autrefois de l'expérience acquise dans les bureaux d'étude, était une démarche pragmatique souvent mise en échec : il manquait en effet des outils permettant de guider le bureau d'étude et de prévoir avec un degré raisonnable de certitude les situations acoustiques futures.

L'analyse acoustique de plusieurs installations hydrauliques réalisées conjointement ou non par les Constructeurs et le Maître d'CEuvre a permis de définir une stratégie mise en œuvre à Electricité de France, dès la conception des ouvrages : le bureau d'étude dispose aujourd'hui d'un guide type dont le caractère opérationnel est démontré par l'excellente qualité des résultats atteints à Saint-Guillerme : tout plancher accessible en permanence par le personnel d'exploitation est affecté d'un niveau sonore inférieur à $85 \mathrm{~dB}$ (A).

Ce résultat est obtenu pour un investissement réduit : $0,4 \%$ du coût de l'usine. Pour parvenir à un bon compromis entre ce surcoût et l'apport réel de l'insonorisation au confort d'utilisation, les différentes zones de la centrale sont considérées chacune avec des objectifs adaptées aux besoins d'exploitation.

Le développement des outils nouveaux de l'acoustique prévisionnelle permet aujourd'hui de prévoir des cartes de bruit dans les usines futures et d'optimiser l'insonorisation des matériels et les traitements à réaliser dans les locaux.

Cet apport récent de la recherche en acoustique doit permettre une meilleure formulation et un meilleur contrôle des spécifications relatives au bruit des machines elles-mêmes, tout en facilitant la prise en compte des aspects contractuels dans la relation entre maître d'œuvre et constructeur.

C'est par l'introduction du facteur " acoustique " dans l'analyse du fonctionnement et de la conception des machines futures, qu'une nouvelle étape vers des usines silencieuses sera franchie : la Société hydrotechnique de France et la Société française d'acoustique* ont décidé de participer à cet effort.

\section{Remerciements}

Les auteurs remercient MM. Bochu, Luzzato, Rio, Taillifet et Tamalet de leur aide amicale et efficace pour la préparation de cet article.

* précédemment nommé " Groupement des acousticiens de langue française ". 


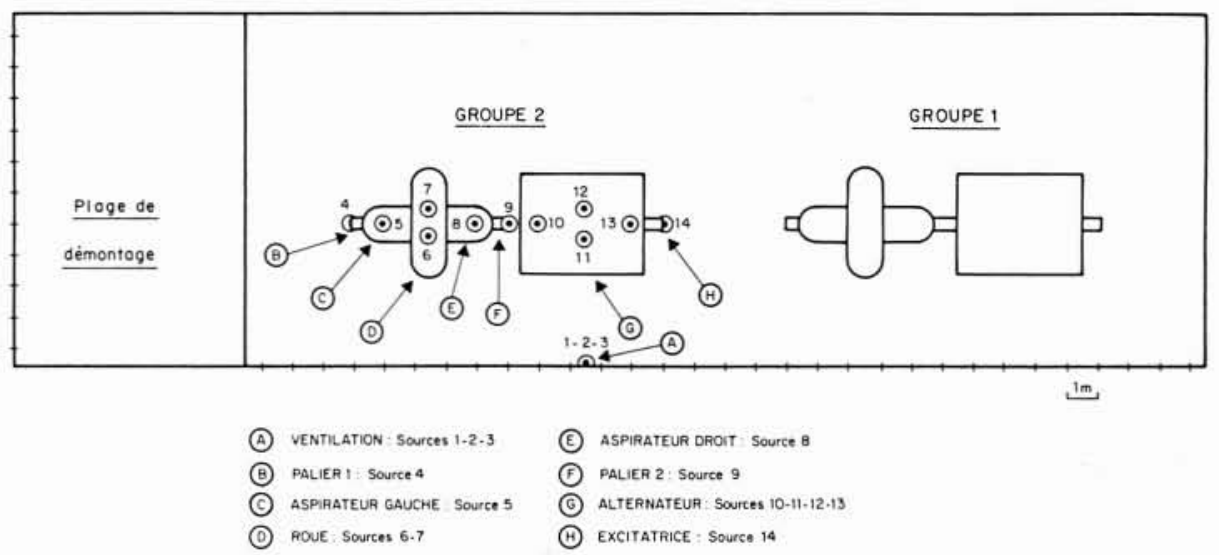

20. Centrale d'Eygliers - Modélisation des sources sonores.

\section{Bibliographie}

[1] Boussuges P., Casacci S., Wegner M. - Bruits associés au fonctionnement de diverses turbomachines. Journées $S H F-G A L F$, décembre 1981.

[2] Détermination de la puissance acoustique émise par les sources de bruits. Norme AFNOR NF S 31067 (Partie 7) : " Méthode de contrôle faisant appel à une source sonore de référence étalonnée sur un plan réfléchissant ". (Janvier 1982).

[3] Aménagements hydrauliques: Etude de la réduction du bruit pour assurer la protection du personnel et du voisinage. Note interne. EDF. DER - 09/1984.

[4] Francois P., Liénard P. - Traité d'acoustique industrielle, Eyrolles ed. Paris 1983.

[5] Barbry J.L., Ondet A.M., Cafaxe M. - Prévision des niveaux sonores dans les locaux comportant des zones d'encombrement différent, $12^{\circ} I C A$, Toronto (1986).

[6] Hamon L., Luzzato E., Nguyen Van Chi, Planchard J. On the use of the finite difference technique to the resolution of the Helmholtz equation in large closed spaces. $12^{\circ} I C A$, Toronto (1986).

[7] Luzzato E., Lecointre C. - Some simple and effective methods for sound source identification with geometrical acoustics models. J. Sound and vibrations (1986), 105.

[8] Degrave C., Taillifet D. - A general method of noise control in a nuclear power plant. Internoise, Cambridge USA (1986). 